\title{
Ewing's sarcoma in pregnancy: a case report
}

\begin{abstract}
Ewing's sarcoma is a primary bone tumour that usually occurs in the first two decades of life. The diagnosis of Ewing's sarcoma in pregnancy is very rare; there are only sixteen cases published in the literature. We present the seventeenth case of a 34 years old woman diagnosed with Ewing's sarcoma at 32 weeks gestation. The patient was treated with chemotherapy after the safe delivery of her healthy baby by caesarean section at 35 weeks gestation. Overall maternal and fetal outcomes are good when treatment is administered promptly and under the care of a multi-disciplinary team.
\end{abstract}

Volume 5 Issue 5 - 2016

Dina Tannous, T Datta, J Amu

Blackpool Victoria Hospital, UK

Correspondence: Dina Tannous, Specialty registrar in

Obstetrics and Gynaecology, Blackpool Victoria Hospital, UK, Tel

78852054 I I,Email dina.tannous@doctors.org.uk

Keywords: Ewing’s sarcoma, Pregnancy

Received: June 18,2016 | Published: November 10, 2016

Abbreviations: MRI, Magnetic resonance imaging; IGF 1, Insulin like growth factor 1; CT, Computed tomography; IUGR, Intra uterine growth restriction; IUD, Intra uterine death

\section{Introduction}

Ewing's sarcoma is a primary bone tumour that usually occurs in childhood and adolescence. It belongs to the Ewing's sarcoma family of tumours; a collective term used to describe a group of primary bone and soft tissue tumours. Its incidence peaks in the second decade of life and its prevalence is 1.9 per million as quoted by The Bone Cancer Research Trust 2010. ${ }^{1}$ Previous reports have described sixteen cases of Ewing's sarcoma diagnosed in pregnancy. Prompt treatment of this aggressive tumour is vital, however, its effect on maternal and fetal health must be balanced. We hereby present the seventeenth case of Ewing's sarcoma in pregnancy.

\section{Case presentation}

A 34-year-old woman presented to the emergency department at 17 weeks gestation with left knee pain. She was in her second pregnancy; had a caesarean section in the past for failure to progress in labour. She had a body mass index of 27 , was a non-smoker and had no pastmedical history.

A left knee x-ray showed an aneurysmal bone cyst over the head of the fibula with evidence of periosteal reaction and possible fracture. An urgent magnetic resonance imaging (MRI) scan was requested and confirmed a mass expanding the fibular shaft (Figure 1). Figure 1 MRI: Tumour mass expanding the shaft of the fibula.

Consequently, the patient was referred to a tertiary centre for biopsy, and histological diagnosis of Ewing's sarcoma was confirmed at 32 weeks gestation. A multidisciplinary team agreed to perform an elective caesarean section at 35 weeks gestation with a view of starting chemotherapy post-partum. Meanwhile, a computed tomography scan (CT) of the chest, abdomen and pelvis ruled out metastasis.

At 34 weeks, a caesarean section was performed after administration of steroids and a healthy baby girl was born, weighing $2.6 \mathrm{~kg}$. One-week post partum the patient was started on chemotherapy; a combination of vincristine, doxorubicin, cyclophosphamide, ifosfamide and etoposide. She received three cycles of treatment followed by radiotherapy. The baby is well at 3 months follow up and the mother is awaiting excisional surgery.

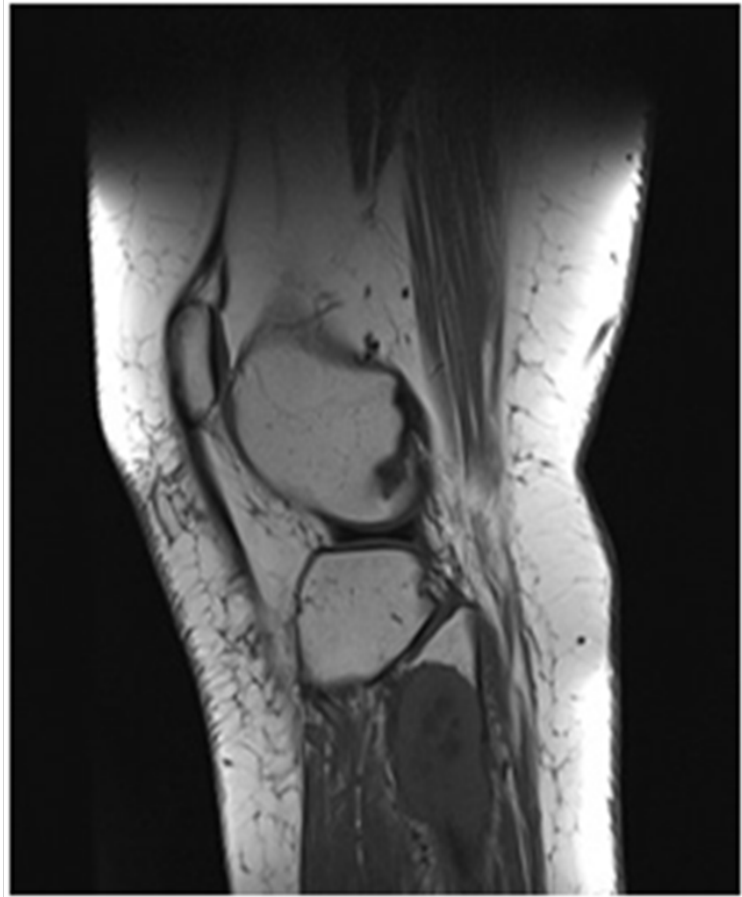

Figure I MRI Tumour mass expanding the shaft of the fibula.

\section{Discussion}

The Ewing's family of tumours is a rare group of bone and soft tissue tumours. Patients often present with persistent pain at the affected site followed by a palpable mass. A common translocation between chromosomes 11 and 22 is identified in approximately $85 \%$ of Ewing's sarcoma cases; t(11;22)(q24;q12) as reported by Iwamoto. ${ }^{2}$

A national case control study of 106 patients by Valery et al. ${ }^{3}$ has raised the possibility of an association between maternal exposure to glandular fever, urinary tract infections during pregnancy and the development of Ewing's sarcoma in the offspring. Due to the rarity of this disease and the small numbers in this study, such claims necessitate further research.

Ewing's sarcoma typically arises within the diaphysial region of the bone. It later invades the bone cortex and surrounding soft tissue. The clinical appearance of the inflammatory reaction surrounding 
the tumour can be confused with osteomyelitis. The gold standard diagnostic test is tissue sampling using fine needle aspiration or biopsy. MRI is useful in establishing the extent of invasion of the tumour and is used to assess the response to treatment.

The diagnosis of Ewing's sarcoma in pregnancy is very rare. Several studies have investigated the hormonal effect of pregnancy on the disease by evaluating tumour expression of oestrogen and progesterone receptors. Du Bios et al. ${ }^{4}$ noted negative immunohistochemical staining for such receptors. On the other hand, Insulin-like growth factor-1 (IGF 1) receptors are almost universally expressed in Ewing's sarcoma cells. This suggests that the increased concentrations of IGF-1 in pregnancy may play a role in expediting recurrence and progression of the tumour.

Chemotherapy is the current treatment of choice in pregnancy and is considered safe in the third trimester. If administered in the first trimester it may cause malformations, intra uterine growth restriction (IUGR) and intra uterine death (IUD). Administration of chemotherapy during the second trimester is accepted and is shown to markedly improve maternal survival when compared to delaying treatment until after delivery.

The use of radiotherapy in pregnancy is dependent on the site and type of tumour. Radiation to the pelvis or nearby sites is best avoided. There is an association between radiation exposure and the development of fetal anomalies, IUGR and IUD particularly if administered in the first trimester. There is a link between maternal exposure to radiation in pregnancy and childhood cancers and leukemia in the offspring. The majority of published cases of Ewing's sarcoma in pregnancy use chemotherapy as the main modality of treatment, however, the use of brachytherapy at the tumour site has been reported by Loguidice. ${ }^{5}$
The effect of the treatment on the mother's longevity of life must be estimated and a decision on timing of delivery can be made. In this case there was a 4 weeks delay from time of diagnosis until administration of treatment. This was due to a delay in liaising with the tertiary center and a patient decision to deliver the baby prior to administering treatment. Overall, diagnosis of primary Ewing's sarcoma in pregnancy has good maternal and fetal outcomes. The best treatment approach is that which is devised promptly with the patient under the guidance of a multi-disciplinary team.

\section{Acknowledgments}

Blackpool Victoria Hospital IT department for providing scan images.

\section{Conflicts of interest}

None.

\section{References}

1. Bone cancer research trust. 2010.

2. Iwamoto, Yukihide. Diagnosis And Treatment Of Ewing's Sarcoma. Japan Journal of Clinical Oncology. 2007;37(2):79-89.

3. Valery PC, McWhirter W, Sleigh A, et al. A National Case-Control Study Of Ewing's Sarcoma Family Of Tumours In Australia. Int J Cancer. 2003;105(6):825-830

4. DuBois SG, Perez-Atayde AR, McLean TW, et al. Late Recurrence Of Ewing Sarcoma During Pregnancy: a report of 2 cases. J Pediatr Hematol Oncol. 2008;30(9):716-718

5. Loguidice V, Tehranzadeh J, Jones E. Ewing's Sarcoma And Pregnancy. Clin Orthop Relat Res. 1986;(210):132-136. 